\title{
Treatment with ginkgo biloba extract protects rats against acute pancreatitis-associated lung injury by modulating alveolar macrophage
}

\author{
Xiao-Wu Xu ${ }^{1,2}$, Xiao-Min Yang ${ }^{3}$, Yong-Heng Bai ${ }^{4}$, Yan-Rong Zhao ${ }^{4}$, Gong-Sheng Shi' ${ }^{2}$, Jian-Guo Zhang ${ }^{2}$, Yi-Hu Zheng ${ }^{4}$ \\ ${ }^{1}$ Department of General Surgery, the Second Affiliated Hospital of Wenzhou Medical University, Wenzhou, China \\ 2Department of Surgery, Affiliated Hospital of Nantong University, Nantong, China \\ ${ }^{3}$ Department of Pathology, the People's Hospital of Wenzhou, Wenzhou, China \\ ${ }^{4}$ Wenzhou Key Laboratory of Surgery, the First Affiliated Hospital of Wenzhou Medical University, Wenzhou, China
}

Prz Gastroenterol 2014; 9 (1): 43-48

DOI: $10.5114 /$ pg.2014.40850

Key words: ginkgo biloba extract, acute pancreatitis, lung injury, alveolar macrophage.

Address for correspondence: $\mathrm{Dr}$ Yi-Hu Zheng, Wenzhou Key Laboratory of Surgery, the First Affiliated Hospital of Wenzhou Medical University, phone: +86 057788288 181, fax: +86 0577 55578999+669385, e-mail: zjsurgery@gmail.com

\begin{abstract}
Introduction: Acute pancreatitis (AP) protease release induces lung parenchymal destruction via inflammatory mediators. Ginkgo biloba has been reported to have anti-inflammatory effects.

Aim: To evaluate the effect of ginkgo biloba extract on experimental acute pancreatitis-associated lung injury in the rat and to investigate the underlying mechanisms.

Material and methods: Acute pancreatitis was induced in rats by injection of $5 \%$ sodium taurocholate into the biliary pancreatic duct. Ginkgo biloba extract (GBE) was administered and pancreas and lung injury were assessed by histological examination. Alveolar macrophages were harvested by bronchoalveolar lavage. Specificity fluorescent probe DAF-FM-DA was applied to observe nitric oxide (NO) bioavailability in alveolar macrophage. The expression of tumour necrosis factor $\alpha$ (TNF- $\alpha$ ) and macrophage migration inhibitory factor (MIF) protein in alveolar macrophage was studied by ELISA.

Results: In sodium taurocholate-induced acute pancreatitis, treatment with GBE significantly protected rats against lung injury associated with pancreatitis in histological sections. Ginkgo biloba extract had a tendency to down-regulate NO bioavailability compared with the AP group, but without statistical significance. Moreover, TNF- $\alpha$ and MIF at protein levels in alveolar macrophage with GBE treatment were decreased compared with the AP group.

Conclusions: These results suggest that GBE could effectively protect rats against acute pancreatitis-associated lung injury. The GBE may inhibit excessive activation of alveolar macrophages from acute pancreatitis-associated lung injury through down-regulation of generation of NO, TNF- $\alpha$ and MIF. These findings suggest that ginkgo biloba extract is a suitable candidate as an effective strategy against acute pancreatitis-associated lung injury.
\end{abstract}

\section{Introduction}

Acute lung injury (ALI) still represents a problem in acute pancreatitis (AP). About one-third of all deaths from acute pancreatitis have been known to occur prior to admission to hospital, and in most cases they are associated with ALI [1]. Acute lung injury is a consequence of excessive leukocyte activation with increased endothelial and epithelial barrier permeability, with leakage of protein-rich exudates into the alveolar space and interstitial tissues, thus compromising oxygenation and gas exchange [2]. It has been reported that alveolar macrophage is involved in the development of acute local disorder as a consequence of extra-pulmonary stimuli like acute pancreatitis, peritonitis or trauma [3]. Alveolar macrophage has the capacity to secrete a vast number of chemokines, cytokines, growth factors and reactive oxygen and nitrogen species. Ginkgo biloba, which is considered a living fossil, has been used for medicinal purposes for thousands of years. Several beneficial biological activities and pharmacologic effects of ginkgo biloba extract (GBE) have been proposed: anti-oxidation, anti-inflammation, anti-tumour, anti-ageing, and cardio-protective properties [4]. 


\section{Aim}

This study aims to observe the model of acute pancreatitis in rats with lung injury and to explore the effect of GBE on the function of alveolar macrophage in acute pancreatitis rats with lung injury.

\section{Material and methods \\ Animals}

A total of 48 male and female Sprague-Dawley rats, each weighing 200-250 g, were obtained from Nantong University Animal Experiment Centre. They were housed in groups with free access to water and standard rat chow diet. Prior to the experiment, rats were fasted overnight. The study protocol was approved by the Animal Care and Use Committee of Nantong University.

\section{Experimental design}

Animals were randomized into three groups: (1) control, (2) acute pancreatitis (AP), and (3) AP plus GBE (Shupei Doctor Pharmaceutical Factory, Weimar, Germany) treatment. Rats in the control group and the two AP groups were subjected to a sham operation and an AP induction procedure respectively. The control group only underwent on-off operation. The AP models from the pancreatitis group were produced by injection of $5 \%$ sodium taurocholate $(2 \mathrm{ml} / \mathrm{kg}$, Sigma) into the biliary pancreatic duct, as described in previous studies. The GBE group, based on AP models, was given GBE intervention. During the preoperative 2 days, the GBE group was given GBE (Ginaton injection $20 \mathrm{mg} / \mathrm{kg}$ ) by subcutaneous injection, twice a day, at the end of the operation, and then GBE was given once. The control group and AP group were given equivalent physiological saline. At 6 and $12 \mathrm{~h}$ after operation, respectively, eight rats from each of the groups were sacrificed. Immediately after the sacrifice, bronchoalveolar lavage was performed. For histological examination and scoring, the entire pancreas and lung were rapidly removed from each rat and fixed in formalin.

\section{Induction of acute pancreatitis}

Rats were fasted overnight before operation and had free access to water. After anaesthetisation by chloral hydrate, the rats were given a midline laparotomy. The AP model of rats was induced by introduction of retro $5 \%$ sodium taurocholate $(2 \mathrm{ml} / \mathrm{kg})$ into the biliary pancreatic duct.

\section{Bronchoalveolar lavage}

Six and twelve hours, respectively, after operation, the rats were put to death. Lungs were exposed after opening the chest. The trachea was exposed and intu- bated with a tracheal cannula. Bronchoalveolar lavage collection was performed by flushing the airways and lungs repeatedly 5 times with sterile saline $(8 \mathrm{ml}$ each time). The pooled bronchoalveolar lavage fluid was collected on ice and centrifuged at $1000 \mathrm{rpm}$ for $10 \mathrm{~min}$ and the cell-free supernatant was abandoned. Cells were rinsed with D - Hanks liquid and counted. Then the cells were suspended in RPMI (Roswell Park Memorial Institute) -1640 containing $10 \%$ calf serum at a density of $1 \times 10^{6} / \mathrm{ml}$ cell suspension and seeded in a 24 -well cell culture plate. Cell viability was measured by the trypan blue test (>95\%) and cell purity was determined by Wright-Giemsa staining (> 90\%).

\section{Histological examination}

Paraffin-embedded pancreas and lung samples were sectioned $(5 \mathrm{~mm})$, stained with haematoxylin and eosin, and examined by an experienced pathologist who was not aware of the sample identity. For these studies, 6 randomly chosen microscopic fields were examined for each tissue sample. The histological evaluation of pancreatic injury was examined and semi-quantified on the basis of the improved Schmidt evaluation criteria [5]. The criteria of histological lung injury score were based on the method of Hofbauer et al. [6].

\section{Measurement of nitric oxide}

\section{bioavailability in alveolar macrophage}

Nitric oxide levels in alveolar macrophage were assessed with 4-amino-5-methyl-amino-2',7'-difluorofluorescein diacetate (DAF-FM-DA, Molecular Probe). The collected alveolar macrophages were washed in HEPES buffer salt solution and loaded with DAF-FM-DA. Subsequently, the alveolar macrophages were rinsed 3 times with phosphate buffered saline (PBS), kept in the dark and maintained at $37^{\circ} \mathrm{C}$ with a warm stage on a laser scanning confocal microscope (Leica, Wetzlar, Germany). Fluorescence images were obtained with a 488-nm (excitation) and 515-nm (emission) filter set for DAF-FMDA. Quantitative analysis was carried out by averaging the peak relative fluorescent intensity (optical density arbitrary units) of each image and normalisation of the fluorescent result by the total number of cultured cells counted from each corresponding digitalised phase contrast microscope image [7].

\section{ELISA Assay}

Alveolar macrophages were seeded in a 24-well cell culture plate. The supernatants were collected and analysed with multiplex cytokine detection systems after 24-hour culture. All methods were carried out using the kits according to the manufacturers' instructions (TNF- $\alpha$ ELISA Kit, Bender Med Systems Company; MIF ELISA Kit, 
Chemicon Company). All data were analysed as recommended by the manufacturers.

\section{Statistical analysis}

All experimental data were summarised as means \pm SD. Differences between groups were tested with an ANOVA with subsequent Scheffe post-hoc test using SPSS software. A value of $p$ of less than 0.01 was considered statistically significant.

\section{Results}

\section{Pathologic changes of pancreas and lung tissues}

There were no obvious pathological changes in the pancreatic tissues of the control group. Histological observations showed obvious acinar cell necrosis and haemorrhage, interstitial oedema and intensive infiltration of inflammatory cells in the AP group. The extent of interstitial oedema, inflammatory cell infiltration, acinar cell vacuolisation and necrosis was decreased in the GBE group compared with the AP group. Lung tissues of the control group had no obvious pathological changes. Dilated small blood vessels in interstitial tissues, and red cell and multiple inflammation cell infiltration in alveolus could be seen in the AP group. These changes were reduced significantly in the GBE group. There were no significant dilated small blood vessels in the GBE group. Fewer red cells, andless inflammatory exudate and multiple inflammation cell infiltration were observed (Figure 1 A). The Schmidt standard was applied to score the pancreatic injury of the experimental
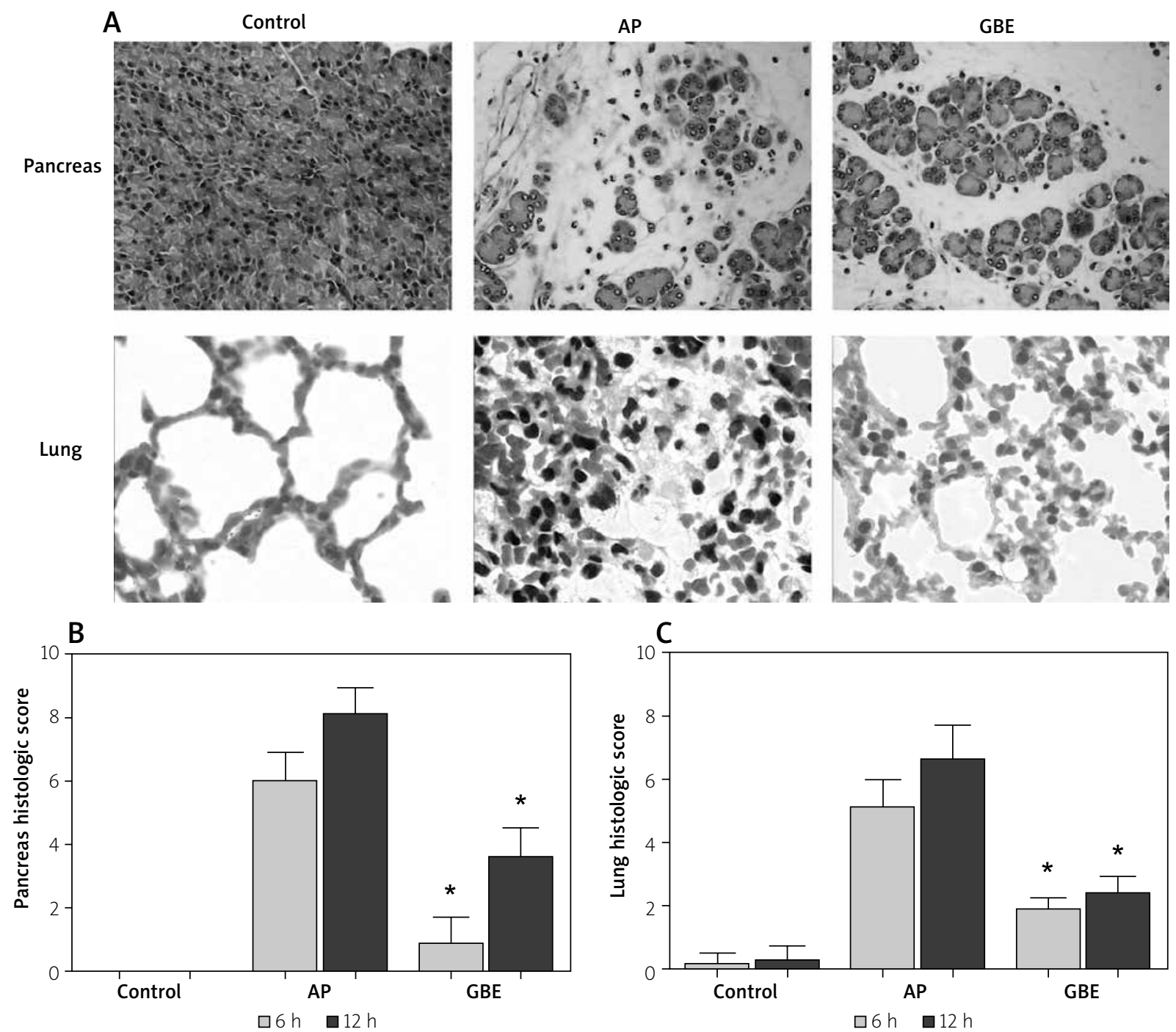

Figure 1. A - H + E staining of pulmonary and pancreatic tissue sections, showing pathologic changes of tissues in the indicated groups $12 \mathrm{~h}$ after operation. B - Pancreas histologic score of rats. ${ }^{*} p<0.01$ vs. AP group. $\mathbf{C}-$ Lung histological score of rats. ${ }^{*} p<0.01$ vs. AP group 
rats from oedema, necrosis, inflammation and haemorrhage. The score system of Hofbauer et al. was applied to score the lung injury of the experimental rats from oedema, inflammatory cell infiltration and haemorrhage. The scores of pancreatic and pulmonary injury are summarised in Figures $1 \mathrm{~B}$ and $1 \mathrm{C}$. At two time points ( 6 and $12 \mathrm{~h}$ ) the injury score for both pancreas and lungs was significantly higher in the AP group than in the control group $(p<0.001)$. The GBE group significantly reduced these scores in the AP animals compared with the AP group $(p<0.001)$.

\section{Nitric oxide bioavailability in alveolar macrophage}

Slight DAF-FM-DA fluorescence was observed in the control group at 6 and $12 \mathrm{~h}$ after the sham operation. A significantly increased cytoplasmic fluorescence of AM was observed in the AP group at 6 and $12 \mathrm{~h}$ af- ter operation compared to the control group ( $6 \mathrm{~h}: p=$ $0.003 ; 12 \mathrm{~h}: p<0.001)$. The AM in the GBE group had decreased the bioavailability in comparison with the AP group at 6 and $12 \mathrm{~h}$, respectively, as shown by the significant and marked reduction in DAF-FM-DA fluorescence (6 h: $p=0.067 ; 12 \mathrm{~h}: p=0.098$ ) (Figure 2).

\section{Tumour necrosis- $\alpha$ and macrophage migration inhibitory factor protein expression}

Acute pancreatitis resulted in a significant up-regulation of TNF- $\alpha$ and MIF protein expression compared with the control group ( $6 \mathrm{~h}: p<0.001 ; 12 \mathrm{~h}: p<0.001)$. Treatment with GBE led to a down-regulation of TNF- $\alpha$ (6 h: $p<0.001 ; 12 \mathrm{~h}: p<0.001)$ and MIF $(6 \mathrm{~h}: p=0.095$; $12 \mathrm{~h}: p<0.001)$ at protein level compared with the AP groups at 6 and $12 \mathrm{~h}$ after operation (Figure 3 ).
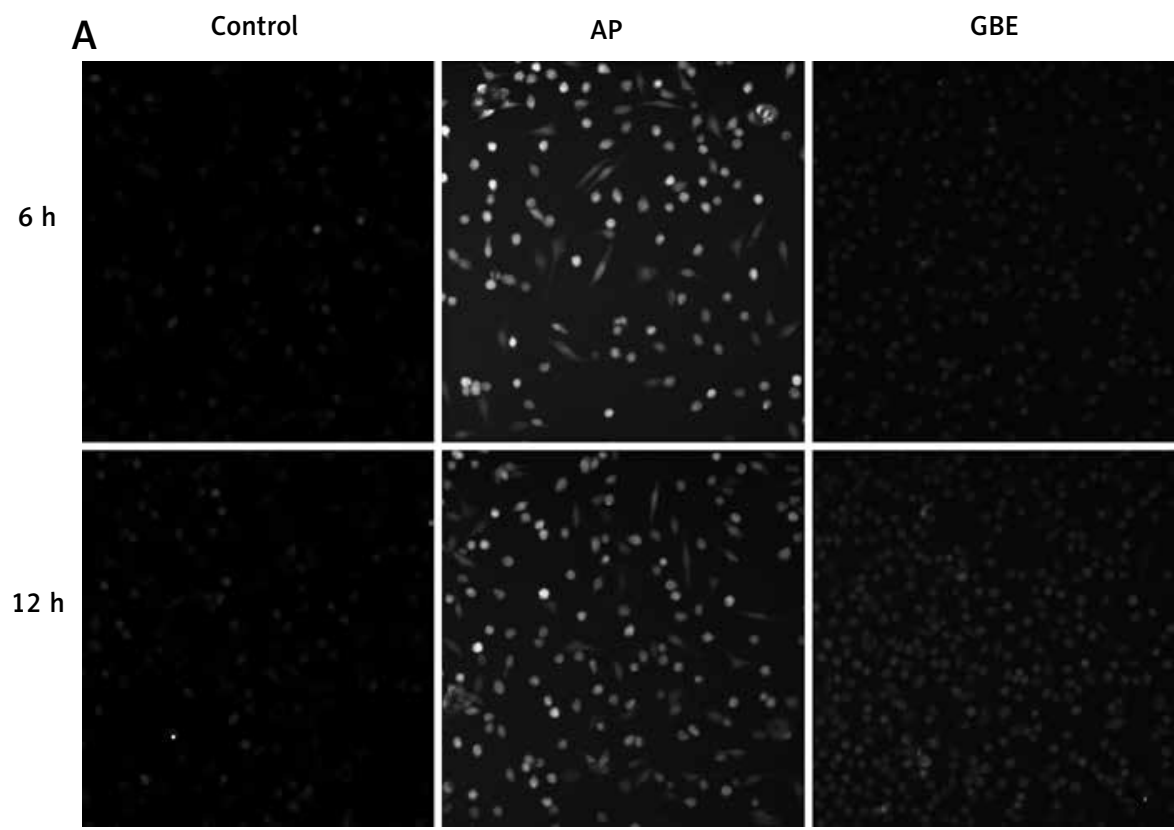

B

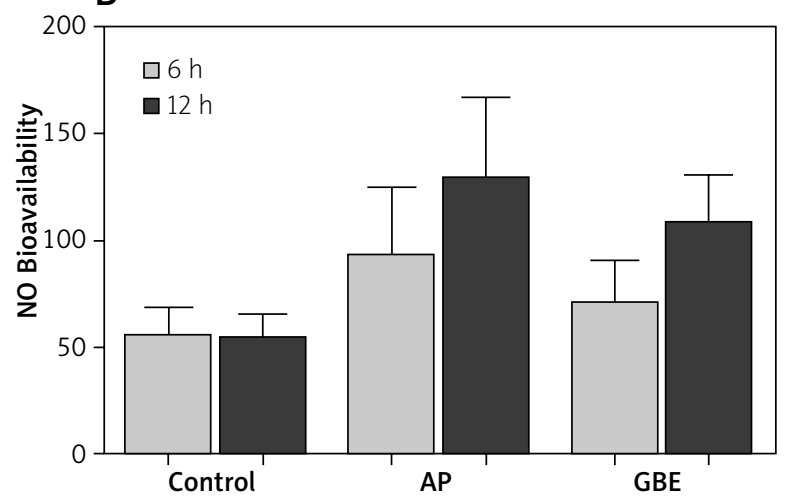

Figure 2. A - Fluorescent detection of NO bioavailability by 4-amino-5-methyl-amino-2', 7' 'difluorofluorescein diacetate (DAF-FM-DA) staining in alveolar macrophage in the indicated groups. B - NO Bioavailability reflected by the fluorescence intensity of DAF-FM-DA 
A

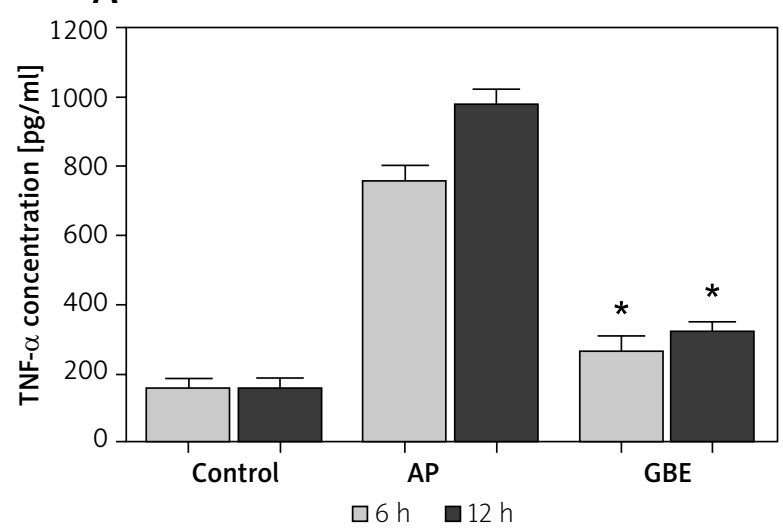

B

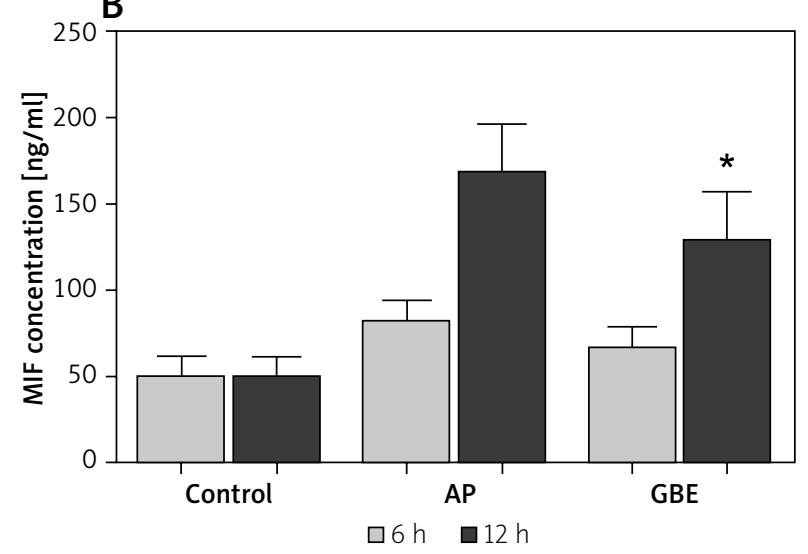

Figure 3. A - The concentration of TNF- $\alpha$ protein expression in the alveolar macrophage. ${ }^{*} p<0.01 \mathrm{VS}$. AP group. B - The concentration of MIF protein expression in the alveolar macrophage. ${ }^{*} p<0.01$ vs. AP group

\section{Discussion}

The development of acute pancreatitis involves a complex cascade of events. The current widely accepted theory is that local injury of the pancreatic acini leads to the leakage of pancreatic enzymes into pancreatic tissue. The leaked enzymes become activated in pancreatic tissues, initiating autodigestion and damage [8].The wild local inflammatory response may lead to pronounced systemic inflammatory response, even distant organ damage and multiple organ dysfunction syndrome.

The ALI and acute respiratory distress syndrome (ARDS) contribute to the majority of AP-associated deaths [9]. Recent research has revealed that alveolar macrophages (AMs) not only serve as phagocytes, but also play an important role as secretory cells to release secretory products such as cytokines, arachidonic acid metabolites and NO in the course of different pulmonary inflammations [10]. Activated AMs can release cytokines and inflammatory mediators which finally result in multiple organ dysfunction syndrome (MODS) [11]. A previous study showed that lung damage induced by experimental AP was associated with alveolar macrophage activation [11].

Nitric oxide represents a potential key mediator of the local and systemic manifestations of AP [12]. Moreover, the role of NO has been increasingly implicated in the pathogenesis of chronic pancreatitis [13]. Sever acute pancreatitis is associated with raised serum NO production in its early stage. Patients with higher serum NO production are at a significantly higher risk of sepsis and mortality [14]. It has previously been shown that NO has a beneficial effect on certain features of acute pancreatitis, both mild and severe [15]. However, a large number of NO production can increase the production of former inflammatory cyto- kines, enhance the expression of endothelial cells and smooth muscle cell adhesion factor, expand the inflammatory reaction, and cause microvascular permeability enhancement and lung damage [16]. Cytokines have been reported to be involved in the development of severe AP. Tumour necrosis factor- $\alpha$ is derived mostly from circulating monocytes and activated fixed-tissue macrophages. Excessive TNF- $\alpha$ production can directly increase pulmonary vascular endothelial permeability and increase procoagulant substance activity, causing lung ischaemia and thrombosis [17]. Macrophage migration inhibitory factor (MIF), a pro-inflammatory cytokine released by activated macrophages, is emerging as an important factor in the pathogenesis of severe acute pancreatitis, and it could restrain macrophage movement and infiltration and secretion of cytokines and inflammation medium in macrophages, which lead to lung injury [18].

In our experimental model, pancreas and lung injury were observed 6 and $12 \mathrm{~h}$ after acute pancreatitis induction. This was reflected by the increased histological pancreas and lung injury scores. In accordance with previous works, the acute pancreatitis model in rats was induced successfully by injection of $5 \%$ sodium taurocholate $(2 \mathrm{ml} / \mathrm{kg})$ into the biliary pancreatic duct. Taking into account the fact that alveolar macrophages may release secretory products (NO, TNF- $\alpha$, MIF) that can act as pro-inflammatory agents, we measured these mediators in macrophages obtained after AP induction. Our results show that these cells are activated as a consequence of acute pancreatitis, resulting in increased generation of NO, TNF- $\alpha$ and MIF. In the present study, although NO, TNF- $\alpha$ and MIF generation by alveolar macrophages were increased after acute pancreatitis induction, GBE could obviously down-regulate these secretory products. These results suggest that GBE 
could effectively inhibit excessive activation of alveolar macrophages from acute pancreatitis-associated lung injury through down-regulation of generation of NO, TNF- $\alpha$ and MIF. Although we suppose treatment with GBE protects rats against acute pancreatitis-associated lung injury from the promising results in animal study, we anticipate a clinical study to validate its potential value in future.

\section{Acknowledgments}

The first author is Dr Xia-Wu Xu and co-first is XioMin Yang.

This article is supported by Wenzhou Municipal SciTech Bureau, Project (No. Y20130097).

\section{References}

1. Zhou MT, Chen CS, Chen BC, et al. Acute lung injury and ARDS in acute pancreatitis: mechanisms and potential intervention. World J Gastroenterol 2010; 16: 2094-9.

2. Shields CJ, Winter DC, Redmond HP. Lung injury in acute pancreatitis: mechanisms, prevention, and therapy. Curr Opin Crit Care 2002; 8: 158-63.

3. Shrivastava P, Bhatia M. Essential role of monocytes and macrophages in the progression of acute pancreatitis. World J Gastroenterol 2010; 16: 3995-4002.

4. Huang $\mathrm{CH}$, Yang ML, Tsai $\mathrm{CH}$, et al. Ginkgo biloba leaves extract (EGb 761) attenuates lipopolysaccharide-induced acute lung injury via inhibition of oxidative stress and NF-kappaB-dependent matrix metalloproteinase-9 pathway. Phytomedicine 2013; 20: 303-9.

5. Schmidt J, Rattner DW, Lewandrowski K, et al. A better model of acute pancreatitis for evaluating therapy. Ann Surg 1992; 215: 44-56.

6. Hofbauer B, Saluja AK, Bhatia M, et al. Effect of recombinant platelet-activating factor acetylhydrolase on two models of experimental acute pancreatitis. Gastroenterology 1998; 115: 1238-47.

7. Gracia-Sancho J, Lavina B, Rodriguez-Vilarrupla A, et al. Increased oxidative stress in cirrhotic rat livers: a potential mechanism contributing to reduced nitric oxide bioavailability. Hepatology 2008; 47: 1248-56.

8. Bhatia M, Wong FL, Cao Y, et al. Pathophysiology of acute pancreatitis. Pancreatology 2005; 5: 132-44.

9. Elder AS, Saccone GT, Dixon DL. Lung injury in acute pancreatitis: mechanisms underlying augmented secondary injury. Pancreatology 2012; 12: 49-56.

10. Dang SC, Jiang DL, Chen M, et al. Clodronate-containing liposomes attenuate lung injury in rats with severe acute pancreatitis. J Zhejiang Univ Sci B 2010; 11: 828-35.

11. Closa D, Sabater L, Fernandez-Cruz L, et al. Activation of alveolar macrophages in lung injury associated with experimental acute pancreatitis is mediated by the liver. Ann Surg 1999; 229: 230-6.

12. Rahman SH, Ammori BJ, Larvin M, et al. Increased nitric oxide excretion in patients with severe acute pancreatitis: evidence of an endotoxin mediated inflammatory response? Gut 2003; 52: 270-4.

13. Morselli-Labate AM, Fantini L, Pezzilli R. Hydrogen sulfide, nitric oxide and a molecular mass $66 \mathrm{u}$ substance in the exhaled breath of chronic pancreatitis patients. Pancreatology 2007; 7 : 497-504.

14. Mettu SR, Wig JD, Khullar M, et al. Efficacy of serum nitric oxide level estimation in assessing the severity of necrotizing pancreatitis. Pancreatology 2003; 3: 506-13.

15. Werner J, Fernandez-del Castillo C, Rivera JA, et al. On the protective mechanisms of nitric oxide in acute pancreatitis. Gut 1998; 43: 401-7.

16. Cheng S, Yan WM, Yang B, et al. A crucial role of nitric oxide in acute lung injury secondary to the acute necrotizing pancreatitis. Hum Exp Toxicol 2010; 29: 329-37.

17. Seo SW, Bae GS, Kim SG, et al. Protective effects of Curcuma longa against cerulein-induced acute pancreatitis and pancreatitis-associated lung injury. Int J Mol Med 2011; 27: 53-61.

18. Matsuda N, Nishihira J, Takahashi Y, et al. Role of macrophage migration inhibitory factor in acute lung injury in mice with acute pancreatitis complicated by endotoxemia. Am J Respir Cell Mol Biol 2006; 35: 198-205.

Received: 18.03 .2013

Accepted: 21.11.2013 